\title{
Speech-Evoked Auditory Brainstem Response in Children with Sensorineural Hearing Loss
}

\author{
Bahram Jalaeia ${ }^{1 *}$ and Mohd Normani Zakariab ${ }^{2}$ \\ ${ }^{1}$ Department of Audiology, Arak School of Rehabilitation, Arak University of Medical Sciences, Markazi, Iran \\ ${ }^{2}$ Audiology and Speech Pathology Programme, School of Health Sciences, University Sains Malaysia, 16150 Kubang Kerian, Kelantan, Malaysia
}

Submission: July 08, 2019; Published: July 22, 2019

*Corresponding author: Bahram Jalaei, Chairman of Audiology Department., School of Rehabilitation, Arak University of Medical Sciences, Markazi, Iran

\begin{abstract}
Speech-evoked auditory brainstem response (speech-ABR) provides an objective documentation of temporal and spectral encoding of speech cues in the brainstem region. As such, the influence of peripheral hearing loss on speech-ABR has been studied but the current literature is limited. The aim of the present study was to compare speech-ABR findings between children with moderate-to-severe SNHL and their agematched normal peers. In this case control study, 17 hearing-impaired (HI) children (mean age $=7.1 \pm 1.8$ years) and 15 typically developing (TD) children (mean age $=6.1 \pm 1.6$ years) were enrolled. All HI children had bilateral moderately severe sensorineural hearing loss. The speechABR was recorded from $\mathrm{HI}$ and TD children to $40-\mathrm{ms}$ speech syllable /da/ presented repetitively at $30 \mathrm{~dB}$ sensation level (SL). Statistically longer latencies were found in HI children than in TD children for onset peaks (V and A) and transition portion (peak C) $(p<0.05)$ with very large effect sizes $(d=1.16-6.10)$. In contrast, the sustained components (peaks D, E and F) and offset portion (peak 0$)$ of speech-ABR were unaffected by hearing loss $(p>0.05)$. For speech-ABR composite onset measures, TD children revealed larger VA inter-peak amplitudes $(d=0.87)$ and steeper VA slopes $(d=1.36)$ compared to HI children $(p<0.05)$. Children with moderately severe sensorineural hearing loss had abnormal encoding of speech sounds within the brainstem region. Based on the study outcomes, the usefulness of speech-ABR can be expanded by studying the speech encoding ability in multi-handicapped children).
\end{abstract}

Keywords: Speech-ABR; Abnormal Encoding; Onset; Sustained; Sensorineural Hearing Loss

Abbreviations: HI: Hearing-Impaired; TD: Typically Developing; ABR: Auditory Brainstem Response; AMLR: Auditory Middle Latency Response; CABR: Cortical Auditory Evoked Potential; OAEs: Otoacoustic Emissions; SD: Standard Deviation; EPSPs: Excitatory Postsynaptic Potentials; EEG: Electroencephalography

\section{Introduction}

In everyday life, people use various speech cues in order to understand ongoing conversations. The acoustical features of speech such as onset time, phoneme transition, periodicity, formant and envelope are encoded and analyzed accordingly by the complex hearing system Skoe \& Kraus [1]. On the other side, previous researches have suggested the notable effects of sensorineural hearing loss (SNHL) on the affected individuals include decreased audibility, poor frequency discrimination, reduced temporal resolution and integration Moore [2]. These negative consequences of SNHL are in fact more prominent in children who are in the process of acquiring speech and language skills Northern \& Downs [3]. Electrophysiological tests offer an objective indication as to how auditory stimuli are processed and encoded at the subcortical and cortical levels Hall [4], Koravand [5], Sharma [6], Skoe \& Kraus [1], Studer-Eichenberger [7]. Auditory brainstem response (ABR), auditory middle latency response (AMLR) and cortical auditory evoked potential (CAEP) are among electrophysiological tests that have been studied in children and adults with auditory dysfunctions Hall [4], Nash Kille \& Sharma [8], Skoe \& Kraus [1], Yang [9].

For many decades, ABR has been used as a standard electrophysiological test for various clinical applications in the fields of audiology and neuro-otology Anwar et al. [10], Hall [1], Koohi [11]. Depending on its specific purposes, ABR is typically recorded with stimuli such as clicks, tone bursts or chirps Birkent et al. [12], Cobb \& Stuart [13], Hall [4], Prendergast et al. [14]. More recently, ABR evoked by complex stimuli has been a subject of increased interest among researchers Koravand [15], Skoe \& Kraus [1]. As such, ABR elicited by complex sounds such as speech syllables can provide supplementary data to the conventional click-evoked ABR and other clinical tests in assessing the neural function Dhar [16], Skoe \& Kraus [1]. When stimulated by a repetitive 40 -ms speech syllable /da/, the speech-evoked auditory brainstem response (speech-ABR) 
waveform typically consists of seven peaks (Figure 1). As shown, peaks $\mathrm{V}$ and A reflect the onset (transient) property of speech$A B R$, that is analogous to wave $V$ of click-evoked ABR Song [17]. These peaks are highly dependent on the neural synchronization upon auditory stimulations Skoe \& Kraus [1]. The consonant-tovowel transition segment of the syllable /da/ is represented by peak C. Peaks D, E, and F represent the phase-locked sustained region (i.e., FFR), while peak 0 reflects the offset portion of steady-state vowel Skoe \& Kraus [1].

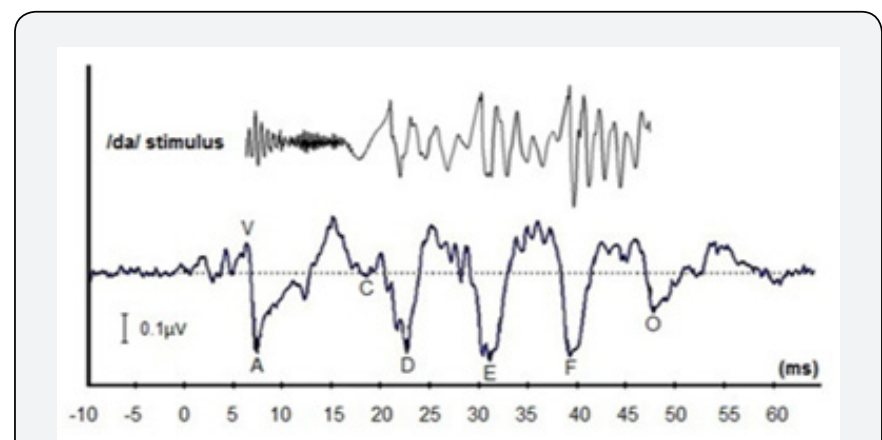

Figure 1: The /da/ stimulus (40-ms duration, upper panel) and the corresponding seven-peak speech-evoked auditory brainstem response of a normal participant (lower panel). Peaks $\mathrm{V}, \mathrm{A}, \mathrm{C}, \mathrm{D}, \mathrm{E}, \mathrm{F}$ and $\mathrm{O}$ are labeled accordingly.

Speech-ABR can be recorded reliably with monaural and binaural stimulations Ahadi [18], Uppunda [19], as well as in the presence of background noise Chandrasekaran [20], Cunningham [21], Hornickel [22]. The responsiveness of speech-ABR in documenting neural plasticity following specific training procedures is also encouraging Jalaei, Filippini [23], Krishnamurti [24], Russo [25], Song [26]. Deficits in the neural encoding of speech cues have been revealed by speechABR in individuals with learning problems Cunningham [21], King [27], Sanfins [28], Wible [29], phonological disorders Gonçalves [30], auditory processing disorders Krishnamurti [24], attention deficit disorders Jafari [31], autism Russo [32], epilepsy Elkabariti [33] and stuttering problems Tahaei [34]. The results of numerous studies on speech-ABR have shown that this electrophysiological response is indeed useful, sensitive and reliable in providing objective documentation of temporal and spectral encoding of complex stimuli Skoe \& Kraus [1]. It can be effectively elicited by stimuli that are transient or sustained, abrupt or slow and continuous or interrupted Skoe \& Kraus [1]. To produce robust speech-ABR waveforms, peripheral hearing system, auditory nerve and brainstem nuclei must be intact and functioning. As such, the influence of peripheral hearing loss on the brainstem processing of speech and complex sounds has been studied but the current literature is limited and contentious. In a study by Koravand [15], speech-ABR results were compared between normally hearing children ( $\mathrm{n}=13$, aged 6 to 14 years) and children with bilateral mild-to-moderately severe SNHL ( $\mathrm{n}=$ 12 , aged 6 to 14 years).

The typical speech syllable /da/ was employed to record the speech-ABR waveforms. While the onset and phoneme transition peaks were unaffected by hearing loss, significantly longer latencies of FFR peaks (D and E) were found in the hearing-impaired group. The authors then concluded that there were specific patterns of subcortical encoding of speech sounds in children with mild-to-moderately severe SNHL. In line with this, Anderson [35] recorded speech-ABR to speech syllable /da/ from normally hearing older adults ( $\mathrm{n}=15$, aged 61 to 68 years) and older adults with mild-to-moderate SNHL $(n=15$, aged 60 to 71 years). They then found that the neural representation of envelope-to-fine structure of the sustained segment of speech sounds was imbalanced in the hearing-impaired group. No such result was seen in the control group. On the other hand, contradictory results were reported by other studies Leite [36], Nada [37]. In a study by Nada [37], speech-ABR (elicited by syllables (/da/ and /ba/) was recorded from 40 adults with bilateral mild-to-moderate SNHL and 20 adults with normal hearing. For both types of speech syllables, statistically longer latencies of speech-ABR onset peaks ( $\mathrm{V}$ and $\mathrm{A}$ ) were found in the hearing-impaired group than in the normal group. When evoked by speech syllable /da/, the hearing-impaired adults also revealed longer peak $\mathrm{C}$ latencies than the normal group.

The sustained FFR features, on the other hand, were not affected by the peripheral hearing loss. The insignificant influence of peripheral hearing loss on FFR peaks was also reported by Leite [36] who studied speech-ABR in children with bilateral mild-to-moderate SNHL. Methodological differences (e.g., different age groups, different severity of hearing loss etc.) are the likely reasons for discrepancies between the results, in which further confirmation is required. Taken together, more research efforts are needed to enhance the current understanding on the influence of hearing loss on the brainstem encoding of speech sounds. As such, speech-ABR outcomes in children with more severe hearing loss have not been reported. The present study, thus, was carried out to compare speech-ABR findings between typically developing children and children with moderately severe SNHL. Due to the notable hearing loss, it was hypothesized that the neural encoding of transient, phoneme transition and sustained segments of speech sounds would be degraded in children with SNHL relative to their age-matched normal peers.

\section{Materials and Methods}

\section{Participants}

Thirty-two Malaysian children aged from 4 to 9 years participated in this case-control study. Of this, 17 of them were hearing-impaired children (mean age $=7.1 \pm 1.8$ years, 8 girls and 9 boys) and the remaining 15 were typically developing children (mean age $=6.1 \pm 1.6$ years, 8 girls and 7 boys). All typically developing (TD) children had no hearing, communication or academic problems and performed normally in pure tone audiometry (PTA thresholds of $\leq 20 \mathrm{~dB}$ HL at 250$8000 \mathrm{~Hz}$ octave frequencies) and tympanometric testing (i.e., 
healthy middle ear function) bilaterally. They were recruited among children of staff members of University Hospital. The hearing-impaired (HI) children, on the other hand, were selected among patients of Audiology Clinic, University Hospital using the convenience sampling method. All of them had hearing levels within a moderately severe range (sensorineural type) with downward sloping patterns bilaterally. They are all hearing aid wearers and had no other medical disabilities (as reported in their medical files). They also had no otoacoustic emissions (OAEs) in both ears, suggestive of poor functions of outer hair cells. All procedures performed in this study involving human participants were in accordance with the ethical standards of Universiti Sains Malaysia (USM/PPP/JEPeM [245.3(5)]) and with the 1975 Helsinki declaration and its later amendments. Prior to the data collection, informed consent was obtained from the parents of children included in the study.

\section{Stimuli and Recording Parameters}

The speech-ABR was recorded according to the standard protocol with a two-channel auditory evoked potential device (Natus Medical Inc., Mundelein, USA). The electrodes were positioned accordingly on each child's head: non-inverting on the vertex, inverting on the right mastoid and ground on the forehead. The impedance of all electrodes was kept below $3 \mathrm{~K} \Omega$ and within $1 \mathrm{~K} \Omega$ of each other. The standard 40-ms syllable / $\mathrm{da} /$ was used as the stimulus to record speech-ABR (Figure 1). It begins with an initial onset burst and followed by a CV transition. The fundamental frequency (F0) of the stimulus rises linearly from 103 to $125 \mathrm{~Hz}$ while the formants shift as follows: F1: $220 \rightarrow 720 \mathrm{~Hz}$; F2: $1700 \rightarrow 1240 \mathrm{~Hz}$; F3: $2580 \rightarrow 2500 \mathrm{~Hz}$. The fourth $(3600 \mathrm{~Hz})$ and fifth $(4500 \mathrm{~Hz})$ formants remain constant for the duration of the stimulus. The stimulus was presented to each child's right ear at $30 \mathrm{~dB}$ SL (i.e., $30 \mathrm{~dB}$ above the averaged hearing level at 500,1000, 2000 and $4000 \mathrm{~Hz}$ ) using the headphones. The stimulus rate was $10.3 / \mathrm{s}$ with 3000 sweeps for each trial. The time window was set at $74.67 \mathrm{~ms}$ (including a $10-\mathrm{ms}$ pre-stimulus period). The acquired responses were amplified 100,000 times and band-pass filtered at 100-2000 Hz. For eliminating the unwanted responses, the artifact rejection was set at $\pm 23.8 \mu \mathrm{V}$. To ensure good waveform replicability, the recording was repeated twice for each trial. During the speech$A B R$ recording, the children laid comfortably in the supine position on the provided bed while watching voiceless cartoons.

\section{Statistical Analyses}

Two experienced audiologists were involved in identifying and verifying speech-ABR peaks. For each child, amplitude and latency values of speech-ABR peaks (V, A, C, D, E, F and O) were computed. Speech-ABR composite onset measures (VA inter-peak duration, VA inter-peak amplitude and VA slope) were also recorded. Since all data were found to be normally distributed ( $p>0.05$ by Kolmogorov-Smirnov normality test), parametric inferential statistical analyses were carried out. To control for the possible effect of age and gender on speech-ABR data, analysis of covariance (ANCOVA, with age and gender as covariates) was conducted to compare the speech-ABR findings between TD and HI groups. Prior to this, the data were initially tested for the homogeneity of slopes to ensure the ANCOVA assumption had not been violated. Pearson analysis was used to determine the correlation between hearing level and speechABR results. The $\mathrm{p}$ values of less than 0.05 were considered statistically significant. In addition, to determine the magnitude of difference between the two groups, Cohen's effect size (d) was computed and interpreted as small $(d=0.20)$, medium $(d$ $=0.50)$, large $(\mathrm{d}=0.80)$ or very large $(\mathrm{d} \geq 1.00)$ Kraemer [38]. All data analyses were conducted with SPSS software version 20 (SPSS Inc, Chicago, IL, USA).

\section{Results}

\section{Age and Hearing Profiles}

In the present study, even though the mean age was descriptively lower in the TD group $(6.1 \pm 1.6$ years $)$ than in the HI group ( $7.1 \pm 1.8$ years), this age difference was not significant $(t=-1.766, d f=30, p=0.088)$. For the HI group, the hearing thresholds ranged from 30 to $85 \mathrm{~dB}$ HL between 500, 1000, 2000 and $4000 \mathrm{~Hz}$ frequencies in both ears. The averaged hearing levels (at 500, 1000, 2000 and $4000 \mathrm{~Hz}$ frequencies) ranged from 51.3 to $71.3 \mathrm{~dB}($ mean $=62.4 \pm 5.7 \mathrm{~dB}$ ) and from 46.3 to $72.5 \mathrm{~dB}$ (mean $=62.9 \pm 7.2 \mathrm{~dB}$ ) for the right and left ears, respectively. These averaged hearing levels were found to be comparable between the ears $(t=-0.372, d f=16, p=0.717)$.

\section{Latency Analyses}

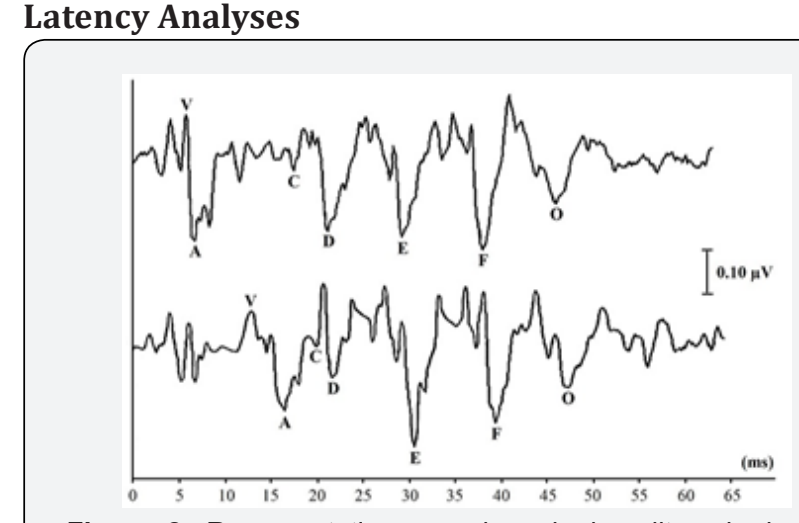

Figure 2: Representative speech-evoked auditory brainstem responses for a typically developing child (upper panel) and a hearing-impaired child (lower panel).

Reliable speech-ABR waveforms had been successfully obtained from all children of both groups. The representative speech-ABR waveforms of a TD child and a HI child are depicted in Figure 2. Mean, standard deviation (SD) and inferential statistical analyses for speech-ABR results for both groups are shown in Table 1. As revealed, the speech-ABR latencies were descriptively longer in the HI group than in the TD group, particularly for onset peaks. This observation was then confirmed by ANCOVA results, in which statistically longer latencies were found in HI children for peaks V, A and C ( $p<0.05)$. For these peaks, effect sizes were very large indicating that substantive 


\section{Global Journal of Otolaryngology}

differences were indeed noted between the two groups $(\mathrm{d}=1.16$ - 6.10). On the other hand, the sustained FFR components (peaks D, E and F) and offset portion (peak O) of speech-ABR were not affected by hearing loss as comparable latency findings were noted between the two groups ( $p>0.05)$. To further support the ANCOVA results, the correlations between hearing level and speech-ABR results were also reported. As shown in Table 2, strong positive correlations were noted between hearing level and latency of peak V $(r=0.90, p<0.001)$ and peak A $(r=0.93$, $\mathrm{p}<0.001$ ). A moderate but significant correlation was found between hearing level and peak $C$ latency $(r=0.54, p=0.001)$. In this regard, latency of these peaks increased with increasing the hearing level. No significant correlations were found between hearing level and latency of sustained peaks of speech-ABR ( $p$ $>0.05$ ).

\section{Amplitude Analyses}

For peak amplitude, the significant statistical result was only found for peak A (Table 1). That is, the mean amplitude of peak A was significantly larger in the TD group $(-0.19 \pm 0.07 \mu \mathrm{V})$ than in the HI group $(-0.07 \pm 0.05 \mu \mathrm{V})(\mathrm{F}=28.374, \mathrm{df}=1,28, \mathrm{p}<0.001)$. This amplitude difference was further supported by a very large effect size $(d=1.99)$. As revealed in Table 2 , a strong correlation was also found between hearing level and peak A amplitude ( $\mathrm{r}$ $=0.71, \mathrm{p}<0.001$ ). No such statistical outcomes were seen for other speech-ABR peaks.

\section{Composite Onset Measures}

While no significant result was found for VA inter-peak duration ( $F=0.131, \mathrm{df}=1,28, \mathrm{p}=0.720)$, TD children revealed statistically larger VA inter-peak amplitudes and steeper VA slopes compared to HI children ( $\mathrm{p}<0.05)$. As shown in Table 1 , the effect size was large for VA slope $(d=0.87)$ and very large for VA inter-peak amplitude $(d=1.36)$. Other than the VA interpeak duration $(r=0.01, p=0.986)$, significant correlations were found between hearing level and composite onset measures of speech-ABR $(\mathrm{p}<0.05)$ (Table 2$)$.

Table 1: Mean, standard deviation (SD), p value for analysis of covariance (ANCOVA, with age and gender as covariates) and Cohen's effect size (d) of speech-evoked auditory brainstem response peak amplitudes, peak latencies and composite onset measures for typically developing (TD) and hearing-impaired (HI) groups.

\begin{tabular}{|c|c|c|c|c|}
\hline & \multicolumn{2}{|c|}{ Mean (SD) } & \multirow{2}{*}{ p value } & \multirow{2}{*}{ Effect size (d) } \\
\hline & $\operatorname{TD}(\mathrm{n}=15)$ & HI $(n=17)$ & & \\
\hline \multicolumn{5}{|l|}{ Latency (ms) } \\
\hline $\mathrm{V}$ & $7.31(0.30)$ & $12.85(1.47)$ & $<0.001 *$ & 5.2 \\
\hline A & $8.40(0.32)$ & $13.88(1.23)$ & $<0.001^{*}$ & 6.1 \\
\hline $\mathrm{C}$ & $19.13(0.93)$ & $20.05(0.65)$ & $0.001^{*}$ & 1.16 \\
\hline $\mathrm{D}$ & $23.15(0.59)$ & $23.21(1.03)$ & 0.856 & 0.07 \\
\hline E & $31.97(0.61)$ & $31.96(1.19)$ & 0.659 & 0.02 \\
\hline $\mathrm{F}$ & $40.57(0.68)$ & $40.86(1.32)$ & 0.795 & 0.28 \\
\hline 0 & $48.99(1.10)$ & $49.86(1.80)$ & 0.138 & 0.58 \\
\hline \multicolumn{5}{|l|}{ Amplitude $(\mu \mathrm{V})$} \\
\hline $\mathrm{V}$ & $0.11(0.05)$ & $0.10(0.08)$ & 0.965 & 0.1 \\
\hline A & $-0.19(0.07)$ & $-0.07(0.05)$ & $<0.001^{*}$ & 1.99 \\
\hline $\mathrm{C}$ & $-0.04(0.05)$ & $-0.06(0.04)$ & 0.117 & 0.58 \\
\hline $\mathrm{D}$ & $-0.17(0.05)$ & $-0.14(0.08)$ & 0.561 & 0.32 \\
\hline E & $-0.21(0.05)$ & $-0.20(0.11)$ & 0.646 & 0.16 \\
\hline $\mathrm{F}$ & $-0.18(0.07)$ & $-0.17(0.11)$ & 0.969 & 0.12 \\
\hline 0 & $-0.12(0.05)$ & $-0.14(0.14)$ & 0.864 & 0.23 \\
\hline \multicolumn{5}{|l|}{ Composite onset measures } \\
\hline VA inter-peak duration (ms) & $1.09(0.01)$ & $1.04(0.60)$ & 0.72 & 0.13 \\
\hline VA inter-peak amplitude $(\mu \mathrm{V})$ & $0.30(0.11)$ & $0.17(0.10)$ & $0.002^{*}$ & 1.36 \\
\hline VA slope $(\mu \mathrm{V} / \mathrm{ms})$ & $-0.28(0.10)$ & $-0.19(0.13)$ & $0.036^{*}$ & 0.87 \\
\hline
\end{tabular}

*Statistically significant at $p<0.05$.

Discussion

Recall that the present study aimed to compare the speechABR findings between typically developing children and children with moderately severe hearing loss. Since OAEs were absent in all tested HI children (suggestive of cochlear damage), the type of hearing loss was likely to be sensory loss. The comparisons between the two groups were considered appropriate as the age and gender distributions were comparable. Furthermore, age and gender influences (if any) were controlled with the use of ANCOVA when speech-ABR results were compared between TD and HI groups. Overall, based on the statistical results, a pronounced influence of hearing loss was observed on some speech-ABR 


\section{Global Journal of Otolaryngology}

peaks. Different from the findings of previous studies, the peak latencies of TD children in the present study were consistently longer. This is not surprising as low stimulation levels (i.e., 30 dB SL) were used to record speech-ABR. Most of studies on speech-ABR among normally hearing participants utilized high intensity levels ( $\geq 80 \mathrm{~dB}$ SPL), which resulted in waveforms with earlier peak latencies Koravand [15], Leite [36], Sanfins [39]. This latency pattern is common and has been consistently demonstrated in many auditory electrophysiological tests Hall [4], Møller [40], Picton [41]. As reported elsewhere, at high

Table 2: Correlation coefficient $(r)$ and corresponding $p$ values when hearing level (in $\mathrm{dB} H \mathrm{H}$ ) is compared with speech-evoked auditory brainstem response results.

\begin{tabular}{|c|c|c|}
\hline & \multicolumn{2}{|c|}{ Hearing level (dB HL) } \\
\hline & $\mathbf{r}$ & $\mathbf{p}$ \\
\hline \multicolumn{3}{|l|}{ Latency (ms) } \\
\hline $\mathrm{V}$ & 0.9 & $<0.001^{*}$ \\
\hline A & 0.93 & $<0.001^{*}$ \\
\hline $\mathrm{C}$ & 0.54 & $0.001^{*}$ \\
\hline $\mathrm{D}$ & 0.03 & 0.853 \\
\hline $\mathrm{E}$ & 0.01 & 0.941 \\
\hline $\mathrm{F}$ & 0.12 & 0.493 \\
\hline 0 & 0.24 & 0.186 \\
\hline \multicolumn{3}{|l|}{ Amplitude $(\mu \mathrm{V})$} \\
\hline $\mathrm{V}$ & 0.06 & 0.783 \\
\hline A & 0.7 & $<0.001^{*}$ \\
\hline $\mathrm{C}$ & 0.22 & 0.22 \\
\hline $\mathrm{D}$ & 0.18 & 0.318 \\
\hline E & 0.08 & 0.617 \\
\hline $\mathrm{F}$ & 0.06 & 0.714 \\
\hline 0 & 0.12 & 0.502 \\
\hline \multicolumn{3}{|l|}{ Composite onset measures } \\
\hline VA inter-peak duration (ms) & 0.01 & 0.986 \\
\hline VA inter-peak amplitude $(\mu \mathrm{V})$ & -0.57 & $<0.001 *$ \\
\hline VA slope $(\mu \mathrm{V} / \mathrm{ms})$ & 0.45 & $0.009^{*}$ \\
\hline
\end{tabular}

*Statistically significant at $p<0.05$.

\section{Onset peak measures of speech-ABR}

In the present study, the latencies of onset peaks ( $\mathrm{V}$ and A) were significantly prolonged in the HI group with very large effect sizes. Moreover, the hearing levels were positively correlated with the onset peak latencies. This suggests that the temporal encoding of stop consonant in the brainstem region is notably degraded in children with SNHL. This abnormal speech encoding might also contribute to the poor ability of HI children in understanding conversations in their daily life. The delayed onset latencies in HI participants have also been reported in the previous studies on speech-ABR Archana [42], Nada [37] and click-evoked ABR Keith \& Greville [43], Strelcyk [44]. In this regard, the use of transient broadband signals would excite all regions of the cochlea Hall [4]. In cases of downward sloping hearing loss (where high-frequency hearing loss is more intensity levels, the neural responses are mostly contributed by the basal part of cochlea Picton [41]. At lower intensity levels, the site of excitation moves toward the apical region of cochlear, resulting in ABR with longer latencies Picton [41]. In addition, the earlier ABR latencies at high stimulation levels may also be contributed by the temporal aspect of excitatory postsynaptic potentials (EPSPs). That is, the time taken for EPSPs to reach thresholds is faster at high intensity levels (thus, shorter latencies) than at low intensities Møller [40]. 
resulted in shorter onset peak latencies. It is generally agreed that as the hearing level increases, the absolute latencies of ABR increases Hall [4], Hyde [46]. It is worth noting that the onset peak latencies of HI children found in Koravand et al.'s study were comparable to normal values. This is not surprising as at high intensity levels, absolute latencies of ABR tend to fall within the normal limits, particularly for audiograms with downward sloping patterns Galambos \& Hecox [47], Gorga [45], Hall [4], Keith \& Greville [43]. In the field of auditory electrophysiology, delayed peak latencies would be typically followed by reduced peak amplitudes when testing participants with specific auditory disorders Aihara [48], Hall [4], Lin [49], Pushpalatha \& Konadath [50].

However, due to the fluctuations in background of electroencephalography (EEG) activity, the peak amplitude is less stable and more variable than the peak latency, particularly for low-amplitude early latency responses Chiappa [51], Edwards [52]. In the present study, reduced amplitude in the HI group was found for peak A. The peak amplitude of ABR represents the sum of action potentials of neurons that are synchronously stimulated by transient auditory stimuli. This neural excitation is degraded in the presence of cochlear pathology, particularly when the basal region of cochlea is damaged Steinhoff [53]. Therefore, significantly lower amplitudes of peak A in HI children found in the present study are consistent with the reduced neural synchronization because of the sensory hearing loss. The significant influence of peripheral hearing loss on the amplitude of speech-ABR onset peak was not revealed in other studies Koravand [15], Nada [36], which could be due to amplitude variability or participants' characteristics (e.g., milder degree of hearing loss).

\section{Composite Onset Measures of Speech-ABR}

The physiological representation of the transient segment of syllable /da/ can be further enlightened by the composite onset measures of speech-ABR including VA inter-peak duration, VA inter-peak amplitude and VA slope. In the present study, significantly lower VA inter-peak amplitudes and less steep VA slopes were noted in HI children than in TD children. Reduced VA inter-peak amplitudes in HI participants were also observed in the study by Leite [36], which are again related to the decreased neural synchronization because of the cochlear damage. As highlighted by Wible [29], the VA slope can be a useful indicator for describing the neural encoding of the onset segment of speech as it captures both the duration and the amplitude of V-to-A transition. Relative to normal children, less steep VA slopes have been consistently noted in children with learning problems Wible [29], Wible [54]. Since HI children were found to have statistically lower VA inter-peak amplitudes, the less steep VA slopes found in this group were in fact sensible. The VA inter-peak duration, on the other hand, was not influenced by the peripheral hearing loss, which is in line with the previous study outcomes Nada [37].

\section{Phoneme Transition Measure of Speech-ABR}

In speech-ABR, the consonant-to-vowel transition segment is represented by peak C. Compared to other speech-ABR peaks, peak $C$ has the smallest amplitude and may not be present in all healthy subjects Skoe \& Kraus [1]. In the present study, nevertheless, the peak $\mathrm{C}$ was present in all tested children. While no significant difference in amplitude was found between the groups, HI children produced statistically longer peak C latencies than TD children. This implies that the encoding of phoneme transition cue is disrupted in children with SNHL, which is consistent with the previous speech-ABR outcome Nada [37]. In view of the aforementioned finding, it is also of interest to know whether there is a link between the deficient phoneme transition encoding (as reflected by the peak C of speech-ABR) and the temporal resolution (i.e., the ability of the auditory system to detect subtle changes or "gaps" between phonemes). Behavioral gap detection tasks are typically performed to measure the temporal resolution Moore [2].

Reduced temporal resolution skills (i.e., higher gap detection thresholds) have been reported in individuals with peripheral hearing loss Fitzgibbons \& Gordon-Salant [55], Glasberg [56], Mishra [57], Moore [2] and auditory processing disorders Boscariol [58], Dias [59], which may underlie their poor speech perception. In a study by Sanju [60], gap detection thresholds and speech-ABR results were compared between young and middle-aged adults with normal hearing. As reported, significant differences in the test results were found between the two age groups. On the other hand, no significant correlations were observed between gap detection thresholds and speech-ABR results (peak V latency and FFR amplitude), suggesting that the auditory stimuli might be encoded differently by the two measures. The peak $\mathrm{C}$ of speech-ABR, however, was not analyzed and discussed in this study. Further research is therefore warranted to determine whether there is a significant relation between the peak $\mathrm{C}$ of speech-ABR and the gap detection ability, particularly when testing participants with compromised auditory functions.

\section{Sustained FFR measures of speech-ABR}

In the speech-ABR testing elicited by the consonant-vowel speech syllable, the sustained FFR peaks (D, E and F) represent the phase-locked neural encoding of the steady state vowel Skoe \& Kraus [1]. While the influence of peripheral hearing loss on the onset peaks of speech-ABR was robustly noted in the present study, no significant differences in FFR results were found between $\mathrm{HI}$ and TD children. The insignificant effect of hearing loss on the sustained peaks of speech-ABR has also been reported in the previous studies Leite [36], Nada [37] and there are several possible reasons for this. First, intact peripheral and central auditory pathways contribute to the robustness of speech-ABR. The onset, severity and type of hearing loss may affect speech-ABR results. All HI children participated in the present study had better hearing thresholds at low frequencies 
(i.e., downward sloping audiometric patterns). Therefore, if compared to the high-frequency consonant perception, the vowel segment of speech syllable would be perceived more clearly and with better audibility, resulting in discernible FFR peaks.

Second, since the transient and sustained segments of the speech syllable might be encoded differently (by distinct neural mechanisms within the brainstem region) Akhoun [61], Hoormann [62], Kraus \& Nicol [63], Russo [64], the influence of peripheral hearing loss on these speech elements would also be different. Russo [64] recorded speech-ABR to a repetitive 40-ms speech syllable /da/ from normally hearing children $(n=38$, aged 8 to 12 years) in quiet and in noisy conditions. In the quiet environment, while significant correlations were found between the onset peaks, the sustained FFR measures were not related to each other and had no correlation with the onset peaks. Moreover, when the speech-ABR was recorded in the presence of noise, the onset responses were severely affected but the FFR features remained unaltered. When recording speech-ABR to speech syllable /ba/ in normally hearing adults, Akhoun [61] found that the onset and FFR features of speech-ABR were affected differently by the intensity level. Taken together, these findings suggest that there are two distinct neural streams (within the same pathway) for encoding the transient and sustained segments of speech sounds Kraus \& Nicol [63]. In the study by Nada [37] that compared speech-ABR results between healthy and SNHL adults, while the latencies of onset peaks were statistically longer in the SNHL group, the FFR results were comparable between the two groups.

They then concluded that the neural phase locking to fundamental frequency and its harmonics was not influenced by the peripheral hearing loss, which is in line with the present study outcomes. Different from the present study outcomes and previous reports Leite [36], Nada [37], Koravand [15] found the latencies of FFR peaks (D and E) to be significantly delayed in hearing-impaired children than in the control group. Other speech-ABR peaks (including peak F of FFR), on the other hand, were not influenced by the hearing loss. Moreover, the amplitude of peak 0 was found to be consistently larger in the hearing-impaired group. The exact reasons for the discrepancies between the results are unknown and further large-scale research is warranted to provide more concrete evidence of these aspects of speech-ABR. In the present study, latencies and amplitudes of peak $\mathrm{O}$ were found to be comparable between TD and HI children. This implies that the neural encoding of the end of vowel is unaffected by hearing loss, which is line with the previous speech-ABR findings Leite [36], Nada [37].

\section{Stimulation level issues}

It is worth noting that the present study recorded speechABR at a similar low sensation level (i.e., $30 \mathrm{~dB}$ SL) as an effort to obtain an "equal" level of stimulation for both TD and HI groups. By considering the maximum output limit of the ABR device (around $100 \mathrm{~dB} \mathrm{nHL}$ ) and the maximum hearing levels of hearing-impaired participants (around $70 \mathrm{~dB} \mathrm{HL}$ ), the stimulation level was therefore set at $30 \mathrm{~dB}$ SL. Previous speechABR studies involving hearing-impaired participants used intensity levels between 80-95 dB sound pressure level (SPL) Abd El-Ghaffar [65], Anderson [35], Leite [36], Koravand [15]. Regarding the intensity level for speech-ABR recording, there is a concern whether the stimulus level should be presented in $\mathrm{dB}$ SPL or in dB SL when testing hearing-impaired individuals. When comparisons are made between normal and hearing-impaired listeners, the stimulus presentation at the same SL may result in higher absolute levels perceived by HI participants, leading to unequal (i.e., better) results Lewis [66]. In a study by Lewis [66] that recorded tone-evoked ABR in normal and mild-to-moderate hearing-impaired adults, significantly earlier wave $V$ latencies were reported in the hearing-impaired group when the tonebursts were presented at similar SLs. In contrast, both groups produced similar wave $\mathrm{V}$ latencies with the use of equivalent SPLs in recording ABR.

The earlier ABR latencies in sensory hearing-impaired individuals when the tone bursts were presented at similar SLs can be explained by several factors including decreased cochlear filter build-up time, reduced cochlear amplifier gain and broadened auditory filters Lewis [66]. In the present study, the stimulus presentation at a similar SL in recording speechABR was considered appropriate to obtain the desired results. Different from Lewis et al.'s study (that used tone bursts to record ABR), the present study recorded speech-ABR using the speech syllable /da/ and thus, the findings would be different. In fact, since the HI children in the present study had greater hearing loss, the latencies of onset peaks were in fact delayed (not earlier) compared to that of the TD group [67-70].

\section{Study Limitations}

The present study is not without limitations. The size of sample was modest and perhaps more favorable outcomes would be obtained if more children could be recruited. Furthermore, the data were gathered from children of a limited age range (4 to 9 years) and different study findings might be obtained if different age groups are tested. In this respect, it is of interest to see how the brainstem encoding of speech cues is affected by hearing loss in younger children $(\leq 3$ years of age) and this is subject to further research [71-75].

\section{Conclusion}

In the present study, the brainstem encoding of speech syllable /da/ was compared between children with moderately severe SNHL and their age-matched normal peers by means of speech-ABR testing. As revealed, the neural encoding of steady-state vowel was unaffected by hearing loss. In contrast, the brainstem encoding of transient and phoneme transition segments of speech syllable was significantly disrupted in HI children. Nevertheless, since the differences in speech-ABR 
results between the two groups can be very large (e.g., effect size of 6.10 for peak A latency), it remains puzzling whether the encoding deficits can also be due to compromised neural functions (conditions similar to learning-impaired children) rather than solely caused by peripheral hearing loss. Further research is therefore warranted to shed light on this issue. The present study findings may also provide insight into essential speech elements that require a "special" focus when developing an effective auditory training protocol for children with moderately severe hearing loss. Moreover, since the sustained FFR components are unaffected by hearing loss, the usefulness of speech-ABR can be further expanded by studying multi-handicapped children (those with peripheral hearing loss and auditory brainstem deficits). For example, it is of interest to know whether speech-ABR is useful as an objective tool in distinguishing between hearing-impaired children, and those with peripheral hearing loss and central auditory deficits (that compromise the encoding of sustained speech segment). This diagnostic aspect of speech-ABR is subject to further research [76-78].

\section{Acknowledgement}

This work was supported by Research University (RU) Grant (1001/PPSK/812114), University Sains Malaysia. The authors were grateful to all children and their respected parents for the ongoing cooperation during the data collection process.

\section{References}

1. Skoe E, Kraus N (2010) Auditory brain stem response to complex sounds: A tutorial. Ear Hear 31(3): 302-324.

2. Moore BC J (1998) Cochlear Hearing Loss. Whurr Publishers Ltd, London, UK.

3. Northern JL, Downs MP (2014) Hearing in Children, sixth ed. San Diego, CA, Plural Publishing, US.

4. Greenberg S (1980) Neural Temporal Coding of Pitch and Vowel Quality: Human Frequency-Following Response Studies of Complex Signals. Los Angeles, CA: Phonetics Laboratory, Department of Linguistics, UCLA. Hall JW (2007). New Handbook of Auditory Evoked Responses. Boston: Pearson.

5. Koravand A, Jutras B, Lassonde B (2013) Auditory event related potentials in children with peripheral hearing loss. Clin Neurophysiol 124(7): 1439-1447

6. Sharma A, Glick H, Deeves E, Duncan E (2015) The P1 biomarker for assessing cortical maturation in pediatric hearing loss: a review. Otorinolaringologia 65(4): 103-114

7. Studer Eichenberger E, Studer Eichenberger F, Koenig T (2016) Statistical learning, syllable processing, and speech production in healthy hearing and hearing-impaired preschool children: a mismatch negativity study. Ear Hear 37: 57-71.

8. Nash Kille A, Sharma A (2014) Inter-trial coherence as a marker of cortical phase synchrony in children with sensorineural hearing loss and auditory neuropathy spectrum disorder fitted with hearing aids and cochlear implants. Clin Neurophysiol 125(7): 1459-1470.

9. Yang FF, Mc Pherson B, Shu H, Xiao Y (2012) Central auditory nervous system dysfunction in infants with non-syndromic cleft lip and/or palate. Int J Pediatr Otorhinolaryngol 76(1): 82-89.
10. Anwar A, Singleton A, Fang Y, Wang B, Shapiro W, et al. (2017) The value of intraoperative EABRs in auditory brainstem implantation. Int J Pediatr Otorhinolaryngol 101: 158-163.

11. Koohi N, Vickers, DA, Lakshmanan R, Chandrashekar H, Werring DJ (2017) Hearing characteristics of stroke patients: prevalence and characteristics of hearing impairment and auditory processing disorders in stroke patients. J Am Acad Audiol 28(6): 491-505.

12. Birkent ÖF, Karlıdağ T, Başar F, Yalçın Ş, Kaygusuz I, et al. (2017) Evaluation of the relationship between the air-bone gap and prolonged $A B R$ latencies in mixed-type hearing loss. J Int Adv Otol 13(1): 88-92.

13. Cobb KM, Stuart A (2016) Neonate auditory brainstem responses to CE-Chirp and CE-Chirp octave band stimuli I: versus click and tone burst stimuli. Ear Hear 37(6): 710-723.

14. Prendergast G, Guest H, Munro KJ, Kluk K, Leger A, et al. (2017) Effects of noise exposure on young adults with normal audiograms I: Electrophysiology. Hear Res 344: 68-81.

15. Koravand A, Al Osman R, Rivest V (2017) Speech-evoked auditory brainstem responses in children with hearing loss. Int J Pediatr Otorhinolaryngol 99: 24-29.

16. Dhar S, Abel R, Hornickel J, Nicol T, Skoe E, et al. (2009) Exploring the relationship between physiological measures of cochlear and brainstem function. Clin Neurophysiol 120(5): 959-966.

17. Song JH, Banai K, Russo NM, Kraus N (2006) On the relationship between speech- and nonspeech-evoked auditory brainstem responses. Audiol Neurootol 11(4): 233-241.

18. Ahadi M, Pourbakht A, Jafari AH, Jalaie S (2014) Effects of stimulus presentation mode and subcortical laterality in speech-evoked auditory brainstem responses. Int J Audiol 53(4): 243-249.

19. Uppunda AK, Bhat J, D'costa PE, Raj M, Kumar K (2015) Binaural interaction component in speech evoked auditory brainstem responses. J Int Adv Otol 11(2): 114-117.

20. Chandrasekaran B, Hornickel J, Skoe E (2009) Context dependent encoding in the human auditory brainstem relates to hearing speech in noise: Implications for developmental dyslexia. Neuron 64(3): 311319.

21. Cunningham J, Nicol T, Zecker SG, Bradlow A, Kraus N (2001) Neurobiologic responses to speech in noise in children with learning problems: Deficits and strategies for improvement. Clin Neurophysiol 112(5): 758-767.

22. Hornickel J, Skoe E, Nicol T, Zecker S, Kraus N (2009) Subcortical differentiation of stop consonants relates to reading and speech-in-noise perception. Proc Natl Acad Sci USA 106(31): 13022-13027.

23. Filippini R, Befi Lopes, DM, Schochat E (2012) Efficacy of auditory training using the auditory brainstem response to complex sounds: auditory processing disorder and specific language impairment. Folia Phoniatr Logop 64(5): 217-226.

24. Krishnamurti S, Forrester J, Rutledge C, Holmes GW (2013) A case study of the changes in the speech-evoked auditory brainstem response associated with auditory training in children with auditory processing disorders. Int J Pediatr Otorhinolaryngol 77(4): 594-604.

25. Russo NM, Nicol TG, Zecker S, Hayes EA, Kraus N (2005) Auditory training improves neural timing in the human brainstem. Behav Brain Res 156(1): 95-103.

26. Song JH, Skoe E, Wong PC, Kraus N (2008) Plasticity in the adult human auditory brainstem following short-term linguistic training. J Cogn Neurosci 20(10): 1892-1902.

27. King C, Warrier CM, Hayes E, Kraus N (2002) Deficits in auditory brainstem pathway encoding of speech sounds in children with learning problems. Neurosci Lett 319(2): 111-115. 


\section{Global Journal of Otolaryngology}

28. Sanfins MD, Borges LR, Ubiali T, Colella Santos MF (2017) Speech auditory brainstem response (speech $\mathrm{ABR}$ ) in the differential diagnosis of scholastic difficulties. Braz J Otorhinolaryngol 83(1): 112-116.

29. Wible B, Nicol T, Kraus N (2004) Atypical brainstem representation of onset and formant structure of speech sounds in children with language-based learning problems. Biol Psychol 67(3): 299-317.

30. Gonçalves IC, Wertzner HF, Samelli AG (2011) Speech and non-speech processing in children with phonological disorders: an electrophysiological study. Clinics (Sao Paulo) 66(2): 293-298.

31. Jafari Z, Malayeri S, Rostami R (2015) Subcortical encoding of speech cues in children with attention deficit hyperactivity disorder. Clin Neurophysiol 126(2): 325-332.

32. Russo N, Nicol T, Trommer B (2009) Brainstem transcription of speech is disrupted in children with autism spectrum disorders. Dev Sci 12(4): 557-567.

33. Elkabariti RH, Khalil LH, Husein R, (2014) Speech evoked auditory brainstem response findings in children with epilepsy. Int J Pediatr Otorhinolaryngol 78(8): 1277-1280.

34. Tahaei AA, Ashayeri H, Pourbakht A, Kamali M (2014) Speech evoked auditory brainstem response in stuttering. Scientifica (Cairo) 2014: 328646

35. Anderson S, Parbery Clark A, White Schwoch T, Drehobl S, Kraus N (2013) Effects of hearing loss on the subcortical representation of speech cues. J Acoust Soc Am 133(5): 3030-3038.

36. Leite RA, Magliaro FCL, Raimundo JC, (2016) Effect of hearing aids use on speech stimulus decoding through speech-evoked ABR. Brazilian Journal of Otorhinolaryngology.

37. Nada NM, Kolkaila EA, Gabr TA, Trandil H, El Mahallawi, et al. (2016) Speech auditory brainstem response audiometry in adults with sensorineural hearing loss. Egyptian Journal of Ear, Nose, Throat and Allied Sciences 17(2): 87-94.

38. Kraemer HC, Morgan G, Leech N, Gliner JA, Vaske JJ (2003) Measures of clinical significance. J Am Acad Child Adolesc Psychiatry 42(12): 15241529.

39. Sanfins MD, Borges LR, Ubiali T, Donadon C, Diniz Hein TA, et al. (2016) Speech-evoked brainstem response in normal adolescent and children speakers of Brazilian Portuguese. Int J Pediatr Otorhinolaryngol 90 12-19.

40. Møller AR (1981) Latency in the ascending auditory pathway determined using continuous sounds: Comparison between transient and envelope latency. Brain Res 207(1): 184-188.

41. Picton TW, Stapells DR, Campbell KB (1981) Auditory evoked potentials from the human cochlea and brainstem. J Otolaryngol Suppl 9: $1-41$.

42. Archana G, Kishan MM, Kumar V (2015) Latency-intensity function of speech evoked auditory brainstem responses in individuals with moderate sensory neural hearing loss. Indian J Otol 21(4): 254-259.

43. Keith WJ, Greville KA (1987) Effects of audiometric configuration on the auditory brainstem response. Ear Hear 8(1): 49-55.

44. Strelcyk O, Christoforidis D, Dau T (2009) Relation between derived-band auditory brainstem response latencies and behavioral frequency selectivity. J Acoust Soc Am 126(4): 1878-1888.

45. Gorga MP, Reiland JK, Beauchaine KA (1985) Auditory brainstem responses in a case of high-frequency conductive hearing loss. J Speech Hear Disord 50: 346-350.

46. Hyde M (1985) Effect of cochlear lesions on the ABR. In J Jacobson (Eds.), The Auditory Brainstem Response. San Diego: College Hill Press pp. 133-146.
47. Galambos R, Hecox KE (1978) Clinical applications of the auditory brain stem response. Otolaryngol Clin North Am 11(3): 709-722.

48. Aihara N, Murakami S, Takemura K (2013) Interaural difference of wave $\mathrm{V}$ predicting postoperative hearing in Gardner-Robertson Class II acoustic neuroma patients. J Neurol Surg B Skull Base 74(5): 274-278.

49. Lin HC, Chou YC, Wang C, Hung LW, Shih CP, et al. (2017) Correlation between auditory brainstem response and hearing prognosis in idiopathic sudden sensorineural hearing loss patients. Auris Nasus Larynx 44(6): 678-684.

50. Pushpalatha, ZV, Konadath S (2016) Auditory brainstem responses for click and CE-chirp stimuli in individuals with and without occupational noise exposure. Noise Health 18(84): 260-265.

51. Chiappa KH, Gladstone KJ, Young RR (1979) Brainstem auditory evoked responses: studies of waveform variations in 50 normal human subjects. Arch Neurol 36(2): 81-87.

52. Edwards RM, Buchwald JS, Tanguay PE (1982) Sources of variability in auditory brain stem evoked potential measures over time. Electroenceph Clin Neurophysiol 53(2): 125-132.

53. Steinhoff HJ, Böhnke F, Janssen T (1988) Click ABR intensity-latency characteristics in diagnosing conductive and cochlear hearing losses. Arch Otorhinolaryngol 245(5): 259-265.

54. Wible B, Nicol T, Kraus N (2005) Correlation between brainstem and cortical auditory processes in normal and language-impaired children. Brain 128(Pt2): 417-423.

55. Fitzgibbons PJ, Gordon Salant S (1987) Minimum stimulus levels for temporal gap resolution in listeners with sensorineural hearing loss. J Acoust Soc Am 81(5): 1542-1545

56. Glasberg BR, Moore BC, Bacon SP (1987) Gap detection and masking in hearing-impaired and normal-hearing subjects. J Acoust Soc Am 81(5): 1546-1556.

57. Mishra SK, Dey R, Davessar JL (2015) Temporal resolution of the normal ear in listeners with unilateral hearing impairment. J Assoc Res Otolaryngol 16(6): 773-782.

58. Boscariol M, Guimarães CA, Hage SR, Garcia VL, Schmutzler KM, et al (2011) Auditory processing disorder in patients with language-learning impairment and correlation with malformation of cortical development. Brain Dev 33(10): 824-831.

59. Dias KZ, Jutras B, Acrani I (2012) Random Gap Detection Test (RGDT) performance of individuals with central auditory processing disorders from 5 to 25 years of age. Int J Pediatr Otorhinolaryngol 76(2): 174178.

60. Sanju H K, Bohra V, Sinha SK (2017) Speech evoked auditory brainstem response and gap detection threshold in middle-aged individual. Eur Arch Otorhinolaryngol 274(4): 2041-2048.

61. Akhoun I, Gallégo S, Moulin A, Ménard M, Veuillet E, et al. (2008) The temporal relationship between speech auditory brainstem responses and the acoustic pattern of the phoneme /ba/ in normal-hearing adults. Clin Neurophysiol 119(4): 922-933.

62. Hoormann J, Falkenstein M, Hohnsbein J (1992) The human frequency-following response (FFR): normal variability and relation to the click-evoked brainstem response. Hear Res 59(2): 179-188.

63. Kraus N, Nicol T (2005) Brainstem origins for cortical 'what' and 'where' pathways in the auditory system. Trends Neurosci 28(4): 176181.

64. Russo N, Nicol T, Musacchia G, Nina Kraus (2004) Brainstem responses to speech syllables. Clin Neurophysiol 115(9): 2021-2030.

65. Abd El Ghaffar NM, El Gharib AM, Kolkaila EA (2017) Speech-evoked auditory brainstem response with ipsilateral noise in adults with unilateral hearing loss. Acta Otolaryngol 138(2): 145-152. 
66. Lewis JD, Kopun J, Neely ST (2015) Tone-burst auditory brainstem response wave $\mathrm{V}$ latencies in normal-hearing and hearing-impaired ears. J Acoust Soc Am 138(5): 3210-3219.

67. Aizawa N, Eggermont JJ (2006) Effects of noise-induced hearing loss at a young age on voice onset time and gap-in-noise representations in adult cat primary auditory cortex. J Assoc Res Otolaryngol 7(1): 71-81.

68. Bones O, Hopkins K, Krishnan A, Plack CJ (2014) Phase locked neural activity in the human brainstem predicts preference for musical consonance. Neuropsychologia 58: 23-32.

69. Galbraith GC, Arbagey PW, Branski R, Comerci N, Rector PM (1995) Intelligible speech encoded in the human brain stem frequency-following response. Neuroreport 6(17): 2363-2367.

70. Jafari Z, Malayeri S, Rostami R (2015) Subcortical encoding of speech cues in children with attention deficit hyperactivity disorder. Clin Neurophysiol 126(2): 325-332.

71. Johnson KL, Nicol T, Zecker SG, Bradlow AR Skoe E et al. (2008) Brainstem encoding of voiced consonant-vowel stop syllables. Clin Neurophysiol 119(11): 2623-2635.

72. Krishnan A (2002) Human frequency-following responses: representation of steady-state synthetic vowels. Hear Res 166(1-2): 192-201.

This work is licensed under Creative Commons Attribution 4.0 License

DOI: 10.19080/GJO.2019.20.556042
73. Krishnan A, Xu Y, Gandour J, Cariani P (2005) Encoding of pitch in the human brainstem is sensitive to language experience. Brain Res Cogn Brain Res 25(1): 161-168.

74. Krishnan A, Swaminathan J, Gandour JT (2009) Experience-dependent enhancement of linguistic pitch representation in the brainstem is not specific to a speech context. J Cogn Neurosci 21(6): 1092-1105.

75. Song JH, Nicol T, Kraus N (2011) Test-retest reliability of the speechevoked auditory brainstem response. Clin Neurophysiol 122(2): 346355 .

76. Shafer VL, Schwartz RG, Martin B (2011) Evidence of deficient central speech processing in children with specific language impairment: The T-complex. Clin Neurophysiol 122(6): 1137-1155.

77. Wong PC, Skoe E, Russo NM, Dees T, Kraus N (2007) Musical experience shapes human brainstem encoding of linguistic pitch patterns. Nat Neurosci 10(4): 420-422.

78. Xu Y, Krishnan A, Gandour JT (2006) Specificity of experience dependent pitch representation in the brainstem. Neuroreport $17(15)$ : 1601-1605.

\section{Your next submission with Juniper Publishers will reach you the below assets}

- Quality Editorial service

- Swift Peer Review

- Reprints availability

- E-prints Service

- Manuscript Podcast for convenient understanding

- Global attainment for your research

- Manuscript accessibility in different formats

( Pdf, E-pub, Full Text, Audio)

- Unceasing customer service

Track the below URL for one-step submission https://juniperpublishers.com/online-submission.php 